\title{
Lipid minor constituents in wines. A biochemical approach in the French paradox
}

\author{
E Fragopoulou' \\ C A Demopoulos² \\ S Antonopoulou' \\ 'Department of Science of Nutrition- \\ Dietetics, Harokopio University, \\ Athens, Greece; ${ }^{2}$ Faculty of Chemistry, \\ National and Kapodistrian University \\ of Athens, Athens, Greece
}

\begin{abstract}
The "French paradox" is the observation that the French suffer a relatively low incidence of coronary heart disease, despite having a diet relatively rich in saturated fats. Several theories have been proposed in order to explain this phenomenon and several debates arose. One of them attributed this phenomenon to the regular and moderate consumption of wine in France. More specific, it is thought that the existence of bioactive compounds in wine could have an effect on the cardiovascular system, preventing or delaying atherosclerosis. The mechanisms mediating these beneficial effects include: low-density lipoprotein oxidation; endothelium function; smooth muscle cells proliferation; platelet aggregation and angiogenesis. Several mediators participate in these pathophysiological mechanisms, among them are plateletactivating factor (PAF) and oxidized phospholipids that play a crucial and essential role in the initiation and the progression of atherogenesis. In this review, apart from the already known and well characterized biological effects of wine bioactive compounds, the co-existence of compounds that could modulate the production and the actions of PAF is highlighted. The existence of bioactive compounds in wine that could reduce PAF production and inhibit its actions may offer a new insight into the well known French paradox and expand the already reported mechanisms by including the inhibition of PAF actions.
\end{abstract}

Keywords: wine, bioactive compounds, lipids, platelet-activating factor, atherosclerosis

\section{Historical background}

The history of wine began in the Neolithic Period ( $8500-4000$ BC). The archaeological evidence, including written references, suggest that the idea of winemaking of the wild grape Vitis vinifera started in Caucasia, then spread to Mesopotamia, Phoenicia, Egypt, Greece and, from Greece, westward. Along with olives and grain, grapes were an important agricultural crop that was vital to sustenance and community development. Even though the composition of wine was not known, the ancient physicians used wine also for medicinal purposes. Among them Hippocrates, one of the most respected physicians, in the fifth century $\mathrm{BC}$, used wine as a tranquilizer, an analgesic, a diuretic, and as carrier for drugs. Moreover, Caesar recommended wine with meals in order to protect his soldiers from gastrointestinal infections. In addition, the treatment of wounds with wine is well documented.

Across the centuries wine has survived and even though its use in ancient medicine was indisputable, nowadays science demands strict evidence and wine has to prove its value for human health again.

\section{Diet and health}

Scientific research in recent decades has provided evidence that supports the idea that diet is an important contributor for human health. There are several different dietary patterns, depending on the culture and the location of each country, some of which promote health and others that increase risk of chronic diseases. However, it is 
widely accepted that there are some common characteristics in the diets that promote health. Between them the most important ones are: the high consumption of vegetables, fruits, legumes, and cereals; moderate consumption of fish, milk, and dairy products, mostly in the form of cheese; low consumption of meat and meat products; low saturated fat and high monounsaturated fat consumption; and the moderate intake of alcohol, mainly in the form of wine with meals. The above opinion is supported by various epidemiological studies that indicate that the consumption of a diet with the aforementioned characteristics is associated with a decrease in the incidence of cardiovascular disease (CVD) including coronary heart disease (CHD) and atherosclerosis. ${ }^{1-4}$

On the other hand, a diet with high fat content was considered an unhealthy diet and emphasis was given to reduce saturated fat, trans fat, cholesterol, and lower low-density lipoprotein (LDL) cholesterol to achieve some health benefits. However, there was later provocative evidence that other dietary constituents can reduce CHD in a manner independent of total cholesterol levels. ${ }^{1,5}$ The first evidences for this hypothesis arose from two notable observations in France and in Greece. In 1819, Samuel Blac observed a high rate of CVD in Irish autopsies and an unexplained low rate of CVD cases in France and Mediterranean countries. From 1958 to 1970 , Ancel Keys ran the first epidemiological study, the Seven Countries Study, which investigated the relationship among nutrition, health, and disease and more specifically, the incidence of CHD in 18 regions in seven countries: Italy, Yugoslavia, the United States, the Netherlands, Japan, Finland, and Greece. The results of the study revealed that serum cholesterol was the most reliable predictor of CHD across cultures. However, there were some exceptions. In Finland, where the highest heart attack rates in all the countries studied were found, rates were greater than predicted by mean cholesterol values alone. In contrast in Crete, the rates of heart attack were actually less than predicted, given the average serum cholesterol levels measured. These unexpected results caused researchers to conclude that elevated serum cholesterol levels, although a strong indicator, do not always predict CHD risk. ${ }^{1,5,6}$ Subsequent investigations into the diets of people living in Finland, Japan, and Greece have led to some of the most interesting hypotheses regarding the epidemiology of heart disease yet proposed. These hypotheses, in turn, formed the foundation of the dietary recommendations that were to be popularized as the Mediterranean diet. Since then this phenomenon has been examined more closely by many investigators. Among them the Lyon and the Indo-Mediterranean diet heart intervention studies examined the effect of either a diet rich in fruit, vegetables, nuts, and a linolenic acid-rich margarine or their usual diet. After two years the intervention diet group in both studies had significantly reduced cardiovascular end points in comparison with the control group. ${ }^{7,8}$ Moreover a Cretan Mediterranean diet adapted to a Western population has reported to protect against CHD much more efficiently than the prudent diet did. ${ }^{9}$ Recently, data from an epidemiology study in Greece, the ATIIKA study, confirmed the beneficial effects of Mediterranean diet. ${ }^{10}$

\section{French paradox}

The term "French paradox" was introduced in 1992 by Renaud and De Lorgeril in order to describe the epidemiological observation that the French suffer a relatively low incidence of coronary heart disease, despite having a diet relatively rich in saturated fats. ${ }^{11}$ However the term could be used for any country, such as Greece, Japan, Spain, and other exceptions, where people traditionally consume a diet rich in saturated fat but show lower than expected CHD mortality rate and yet the classic CHD risk factors are the same as in other industrialized countries.

\section{Explanations for the French paradox}

The existence of the French paradox, however, does not establish a cause/effect relationship. The scientific community is still trying to explain the above observation and several debates arise. Several epidemiological studies, clinical trials, and in vitro experiments have been performed in order to provide an explanation for this paradox. It is out of the scope of the present review to get into a detailed report of these studies. However a brief report of the most important hypotheses will be presented.

\section{Underestimation of CHD mortality}

One of the main explanations that were proposed for the French paradox was based on a methodology problem. According to this hypothesis, French physicians, in absence of autopsy data, may not declare all CHD deaths as CHD but as deaths from unknown or unspecified causes and therefore an underestimated CHD mortality may have occurred. ${ }^{12}$ However, even though after the correction for this bias, the results concerning CHD attack and mortality rates show that France is still at a low risk for CHD. Therefore, the underestimation theory could not explain the France paradox.

\section{Time lag theory}

This hypothesis was based in the observation that animal fat consumption and serum cholesterol concentration have 
been similar in France and Britain for a relatively short time: about 15 years. For the decades up to 1970, France had lower animal fat consumption and serum cholesterol and only between 1970 and 1980 did French values increase to meet those in Britain. So this theory supports the idea that the difference is due to the lack of detection of the time lag between increases in consumption of animal fat and serum cholesterol concentrations and the resulting increase in mortality from heart disease. ${ }^{13}$ This hypothesis has caused a big scientific debate, but it has not been widely accepted since it was characterized as a simplified explanation that rests on a small number of well established coronary risk factors and ignores other factors that may play a role.

\section{Mediterranean diet}

One reasonable explanation for the French paradox could be that the overall dietary habits of French people are similar to Mediterranean countries. However France is not considered to be a Mediterranean country and the typical French diet does not have the characteristics of the typical Mediterranean diet. For example, the consumption of olive oil is so low that it is not even reported while butter and beef consumption is higher than in any northern country. Moreover, the French consume the lowest quantities of cereals, rice, fruits, pulses, and nuts among Europeans. Therefore, taking into account the aforementioned data it is unlikely that French are protected against CHD because they follow a Mediterranean diet. ${ }^{14}$ However, other characteristics of a typical French diet may contribute to the explanation of the paradox.

\section{Alcohol consumption}

The first observations were for the association between alcohol consumption and the low incidence of CHD. These reports followed the observation of St Legar and colleagues that drew attention to the inverse relationship between red wine consumption and mortality from ischemic heart disease in 18 different European and American countries. ${ }^{15}$ Based on the findings of the Monitoring System for Cardiovascular Disease (MONICA) project, a worldwide program organized by the World Health Organization, the investigators observed a lower CAD mortality rate in France compared with that in the United Kingdom and the United States, despite the high consumption of saturated fats and similar serum cholesterol concentrations. ${ }^{9,16,17}$ In addition, other risk factors such as blood pressure, body mass index, and cigarette smoking were equivalent in France with those in other industrialized countries. Since the consumption of alcohol and especially wine was much higher in France than in most Western countries, the investigators concluded that the drinking habits of the French people protect them from CAD.

Nevertheless, since then several epidemiological and clinical trials were performed in order to confirm: a) the superiority of wine versus other alcoholic beverages; b) the superiority of red wine versus white wine; and c) the pattern of drinking that promotes health. Although the results of many studies are contradicted, ${ }^{18-26}$ it should be mentioned that the majority of the studies confirmed the superiority of wine in relation with other alcoholic beverages. ${ }^{20-26}$ However, we should bear in mind that grape variation is very important for the biological benefits of the wine (grape variety, fermentation, blend, and aging $)^{27-29}$ and in addition, moderate and regular alcohol consumption promotes health benefits in contrast to "binge" drinking.

\section{Mechanisms of wine protection}

So taking all the reports in account, we concluded that wine contains a number of components other than ethanol that could have an effect on the cardiovascular system and prevents or delays atherosclerosis. In order to understand the effects of wine components in the atherosclerotic process, a brief review in the steps of its development is necessary.

\section{Development of atherosclerosis}

Atherosclerosis is a multifactorial disease process that represents the underling cause of many CVDs. Inflammation, oxidative stress, and thrombosis underlie the onset and the perpetuation of atherosclerosis. Among other pro-inflammatory mediators, platelet-activating factor (PAF; 1-O-alkyl-2-acetyl-sn-glycero-3-phosphocholine) $)^{30}$ and oxidized phospholipids are believed to play a crucial role in the initiation and prolongation of the atherosclerotic lesion. ${ }^{31}$ The levels of PAF are under strict metabolic control (see below) and its action is mediated through specific PAF-receptor (PAFR), which is a member of the seven-transmembranedomain-receptor family coupled to G-proteins. ${ }^{32}$

The process of atherosclerotic lesion could be classified in the following essential steps: a) endothelial dysfunction; b) infiltration of LDL particles as well as circulating leukocytes into the subendothelium; c) LDL oxidation; d) monocytederived macrophages acquire the phenotype of foam cells; e) smooth muscle cells (SMC) migration and proliferation in the subendothelium with matrix synthesis; and f) structural endothelial lesion followed by platelet deposition and thrombus formation (Figure 1).

PAF and oxidized phospholipids levels in the blood can be increased under pathophysiological conditions. 
Deficiency of ingested or endogenous antioxidants could lead in LDL oxidation and peroxidation of cellular membranes phospholipids. The oxidation of LDL (ox-LDL) particles results in increased levels of PAF and oxidized phospholipids with PAF-like activity. ${ }^{33,34}$ Extensive experimental work has shown that atherogenic activities of ox-LDLs can be attributed to these lipids. Moreover, the lipoprotein-associated phospholipase $\mathrm{A}_{2}\left(\mathrm{LpPLA}_{2}\right)$ - the enzyme responsible for the degradation of these lipids - is inactive in ox-LDL particles, resulting in increased levels of PAF and oxidized phospholipids. ${ }^{35}$ Both PAF and PAF-like lipids induce release of active oxygen species ${ }^{36,37}$ that lead to further LDL oxidation. ${ }^{38}$ Therefore, a loop between the oxidation of LDL and atherogenic phospholipids is formed.

Endothelium modulates the microenvironment homeostasis by affecting the flow of macromolecules and cells from the bloodstream to the tissue. The molecules involved in the control of such flow are soluble mediators, surface receptors that translate external signals, and adhesion molecules. In this setting, PAF acts as an autocrine ${ }^{39}$ and paracrine mediator that may modulate endothelial functions. Several stimuli are capable of inducing the synthesis of PAF from endothelium including thrombin, vasoactive mediators, and proinflammatory cytokines, suggesting that PAF may transduce or amplify the signals delivered by these mediators.

PAF activity triggers inflammatory response in endothelial cells and at the same time activates blood cells. Increased PAF activity in blood can initiate rapid inflammatory response in endothelial cells through recognition of its receptor. This results to increased permeability of the endothelium, ${ }^{40-42}$ which is a crucial event in the initiation of atherosclerosis. Subsequently activated endothelial cells rapidly produce and display PAF as well as P-selectin on their cell surface. Cytokines, such as tumor necrosis factor- $\alpha$ (TNF- $\alpha$ ) and interleukin-1 (IL-1), promote the neosynthesis of E-selectin and PAF. ${ }^{43}$ Although E-selectin alone is unable to initiate $\mathrm{Ca}^{2+}$ signaling, it prolongs the actions of PAF, including the calcium influx in adhering neutrophils that enhance their response to the IL- 8 produced by endothelial cells. ${ }^{44,45}$ The presence of PAF on endothelial surface activates neutrophils and monocytes through interaction with its specific receptor on their plasma membranes. Activated blood monocytes express P-selectin glycoprotein ligand-1 (PSGL-1) and adhere rapidly to activated platelets or endothelial cells that display P-selectin. ${ }^{46}$ Monocytes adherent to P-selectin respond to PAF with enhanced activation of nuclear factor- $\kappa \mathrm{B}$ $(\mathrm{NF}-\kappa \mathrm{B})$ and dramatically increased synthesis of monocyte chemoattractant protein-1 (MCP-1), IL-8, TNF- $\alpha$, and other inflammatory gene products. ${ }^{47}$ Blood neutrophils activated by PAF induced "inside-out" signaling of $\beta 2$ (CD11/CD18) integrins, with subsequent adhesion and aggregation, priming for enhanced inflammatory responses, polarization and directional migration, degranulation, and oxygen radical generation. ${ }^{46} \mathrm{PAF}$ is also capable to induce activation and aggregation of platelets. ${ }^{48,49}$ Activated platelets by PAF or other aggregating agonist synthesize PAF that remains cell associated. Subsequently, PAF stimulates a tethering and juxtacrine signaling system at the platelet surface which mediates rolling and tight adhesion of neutrophils through interaction of Mac-1 (CD11b/CD18) with fibrinogen-bound $\alpha \operatorname{IIb} \beta 3 .{ }^{46}$

Therefore PAF and various factors (cytokines, selectins, and integrins) released upon cell activation chemoattract blood cells on the endothelium and initiate the atherosclerotic process. The process is amplified through positive feedback control from PAF and other pro-inflammatory mediators. Selectins mediate initial interaction of blood cells with the endothelium while PAF stimulates integrins into action leading to firm adhesion between blood cells and vascular endothelium. Monocytes move into the subendothelial space and differentiate into macrophages. Ox-LDLs undergo irregular scavenger receptor-mediated uptake by macrophages to form foam cells. Progression of the disease leads to "fatty streaks" and then to "fibrous plaques" formation.

\section{Alcohol}

Ethanol is present in most wines in a percentage of $12 \%-15 \%$ of the total volume and exerts some very well defined protected effects, already mentioned in excellent reviews which are out of the scope of the present review. Briefly, these effects include the antiatherogenic alterations in plasma lipoproteins, the antithrombotic modifications of blood platelet function, as well as of the coagulation and fibrinolysis balance. ${ }^{50}$ However the benefits of moderate alcohol consumption can only partly explain the protective effects of wine.

\section{Bioactive compounds in wine}

Apart from ethanol, there are some other constituents in lower concentrations in wine that also exert protective effects. To these bioactive compounds is attributed the extra effect of wine versus other alcoholic beverages. In recent decades, an effort was made to isolate and identify the biologically active compounds that are responsible for this protection as well as to elucidate the mechanism of their action. The main category of these compounds in wine is the phenolic 
ones that contain a wide range of molecules ${ }^{51}$ with desirable biological properties. Among them are phenolic acids (p-coumaric, cinnamic, caffeic, gentisic, ferulic, and vanillic acids), trihydroxy stilbenes (resveratrol and polydatin), and flavonoids (catechin, epicatechin, and quercetin) while their polymerization gives rise to the viniferins and procyanidins. Red wines are usually made in contact with grape skins, in contrast with white wines that are usually made with free-running juices, having no contact with the grape skins. This is the main reason why the concentration of phenolic compounds in white wines is lower than that of red ones.

Initially, the protective effect of bioactive compounds in wine was attributed to their antioxidant properties, but nowadays it is general accepted that they also exert potent anti-inflammatory actions. There are already outstanding reviews about the protective actions of bioactive compounds in wine. ${ }^{52,53}$ In this review, emphasis will be given in their interaction with PAF and PAF-like lipids production and actions.

\section{LDL oxidation}

As mentioned before, the oxidized phospholipids and PAF generated during LDL oxidation or within oxidatively stressed cells, are the triggers for many of the events observed in developing lesions. Phenolic compounds could protect LDL from oxidation, and therefore reduce the amount of PAF and oxidized phospholipids, through the following mechanisms: a) they could act as a free radical scavenger and react as reducing agents of hydrogen atom-donating molecules; b) they could chelate transition metal ions, which diminishes the capacity of the metal to generate free radicals; c) they could protect from oxidation by endogenous antioxidants such as vitamin $\mathrm{E}$ and carotenoids in the LDL particle, resulting in increased levels and subsequent enhanced antioxidant effects; or d) by altering the activity of several enzymes like serum paraoxonase that hydrolyse lipid peroxides of arterial cells and/or LDL associated or through inhibition of cellular oxygenases such as NADPH oxidase, 15-lipoxygenase, cytochrome $\mathrm{p} 450$, and myeloperoxidase..$^{52,53}$ The difference in total phenolic content between white and red wines as mentioned above was the cause for the hypothesis that red and not white wines had significant antioxidant properties. However, during recent years it became clear that variations in the concentration of these constituents among red wines may be responsible for the range of antioxidant potential exhibited by different red wines ${ }^{54}$ and that there are also some white wines which could have antioxidant abilities similar to those of red wines. ${ }^{55-57}$

\section{Vascular endothelium function}

Among the factors contributing to atherosclerosis is the dysfunction of the vascular endothelial cells. Under physiological situations vascular endothelium produces and releases nitric oxide (NO), which in turns, promotes vasorelaxation, reduces platelet aggregation, and limits the flux of atherogenic plasma proteins into the artery wall. The NO vasorelaxant effect is mediated through the activation of guanyl cyclase leading to the accumulation of cGMP. ${ }^{58}$ A narrow range of NO levels mainly regulated by endothelial NO synthase (eNOS) is required in order to maintain the endothelial barrier while NO levels out of this range increase endothelial permeability. ${ }^{59}$ In addition, NO is believed to be implicated in endothelial PAF production. ${ }^{60}$ PAF increases endothelial permeability by inducing eNOS internalization via caveolae and increasing NO production in human endothelial cells. ${ }^{42,61}$ The NO cofactor, vascular endothelial growth factor also increases permeability while PAF is the direct inflammatory mediator of VEGF proinflammatory activities. ${ }^{62} \mathrm{VEGF}$ released from vascular smooth muscle cells (VSMC) is also a powerful endothelial mitogen and stimulates the expression of adhesion molecules and monocyte chemotactic protein-1. ${ }^{63}$ Red and white wines administered to cholesterol-fed rabbits inhibited the expression of monocyte chemotactic protein-1 in aorta. ${ }^{64}$

A plethora of studies has shown the capability of red wine to induce NO production. The vasodilatatory effect of wine seems to be correlated to the type of phenols. More specific monomeric catechins and simple phenols (benzoic acid, gallic acid, and hydroxycinnamic acids) have no effect while anthocyanins oligomeric proanthocyanidins exert effect. ${ }^{53,65}$ However, white wine rich in tyrosol and hydroxytyrosol as well as resveratrol can upregulate eNOS expression and consequently increase eNOS-derived NO production. ${ }^{66,67}$ Therefore vasodilatatory effect does not apply to all wines and is a function of the variability of wine constituents according to grape varieties, area of cultivation, and vinification methods.

Enhanced leukocyte adhesion is also a crucial step in the development of the lesion. Resveratrol prevents PAF-induced leukocyte recruitment and endothelial barrier disruption in rat ischemia/reperfusion-induced tissue injury. ${ }^{68}$ Moreover, several phenolics such as resveratrol, quercetin and methylgallic acid (a major metabolite of gallic acid) and gallates (gallic acid esters) inhibit TNF- $\alpha$ and/or thrombin and/or IL-1 activation of endothelial cells. ${ }^{69-72}$ In turn, this effect could result to reduce PAF levels since TNF- $\alpha$, IL-1, and thrombin induce endothelial PAF production. ${ }^{73}$ Flavonoid, quercetin 


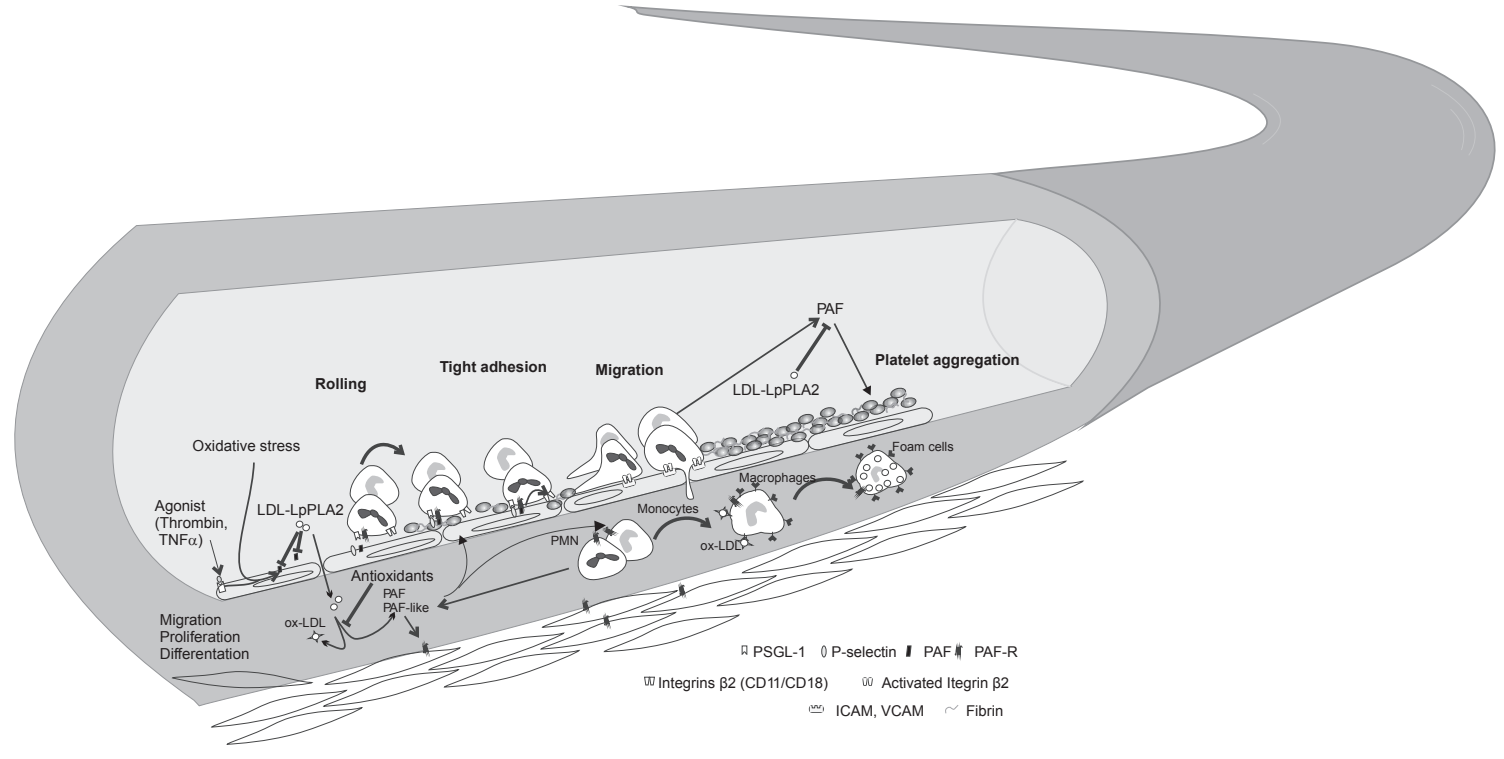

Figure I Essential steps of atherosclerotic lesion and the implication of platelet-activating factor.

Abbreviations: ICAM, intercellular adhesion molecule; LDL, low-density lipoprotein; LPPLA, lipoprotein-associated phospholipase $A_{2}$; ox-LDL, oxidized LDL; PAF, plateletactivating factor; PAF-R, PAF-receptor; PMN, human polymorphonuclear granulocytes; PSGL-I, P-selectin glycoprotein ligand-I; TNF- $\alpha$, tumor necrosis factor- $\alpha$; VCAM, vascular cell adhesion molecule.

luteolin, and apigenin prevent endothelial expression of inducible adhesion molecules and endothelial uptake of ox-LDL. ${ }^{74}$

\section{Vascular smooth muscle cells}

Since the abnormal proliferation and the migration of VSMCs in the arterial intima is a key step in the formation of the atherosclerotic plaque, the inhibition of these processes should retard or inhibit the appearance of arteriosclerosis. It is reported that red wine and its polyphenols inhibit SMC proliferation in a dose-dependent manner. ${ }^{75}$ Platelet-derived growth factor (PDGF) is among the most potent mitogenic and chemotactic agents for VSMC and is released by platelets, endothelial cells, as well as VSMC themselves. PDGF induces cellular motility through activation of the phosphatidylinositol 3-kinase (PI3-K) and p38MAPK pathways while the downregulation of cyclin A gene expression is implicated in the antiproliferation activity of SMC. ${ }^{76-77}$ PAF is also a mitogen for VSMC that has synergistic action with PDGF in this effect ${ }^{78}$ and the presence of functional PAF receptor (PAF-R) in arterial subset SMCs may be important for their migration from the media into the intima and atherosclerotic plaques formation. ${ }^{79,80}$ Ox-LDL also stimulates the proliferation of VSMC and antagonists of PAF-R blocked this effect. ${ }^{81,82}$ The above action of ox-LDL is reported to be due to activation of autocrine or paracrine PDGF loops. ${ }^{83}$ Red wine polyphenols have been shown to inhibit proliferation and SMC migration through downregulation of cyclin A gene expression and inhibition of the PI3-K and p38MAPK pathways. ${ }^{84,85}$ In addition, red wine phenolics reduced the overexpression of VEGF induced by PDGF and other growth factors such as thrombin and transforming growth factor-1 by preventing the redoxsensitive activation of the p38 MAPK pathway in VSMC. ${ }^{86}$

\section{Platelet aggregation}

Many factors could activate platelets including thrombin, ADP, TXA 2 , PAF and many pro-inflammatory mediators, such as PAF, serotonin, transforming growth factor, PDGF, and lipoxygenase metabolites, could be produced by activated platelets, which lead to the progression of atherosclerotic lesions. A lot of studies have investigated the effect of flavonoids on platelet activation and aggregation against several agonists like ADP and thrombin..$^{87-90}$ Our studies have showed that resveratrol and tyrosol inhibit PAF-induced platelet aggregation and that their acetylated derivatives exhibit higher or the same action..$^{91,92}$ The flavonoids fisetin, kaempferol, morin, quercetin and myricetin are able to inhibit washed rabbit platelet aggregation induced by PAF and other agonists. ${ }^{93}$ Several studies have demonstrated that wine or wine extracts are also able to inhibit platelet aggregation significantly. ${ }^{11,94-96}$ Some of these studies presented data that red and white wines have similar effects on platelet aggregation. In this point of view, our team examined the effect of several red and white wines of Greek origin on PAF-induced platelet aggregation. ${ }^{97}$ The total lipid extracts of each were 
separated to neutral and polar lipid extracts. In all wines tested, the polar extract was the most potent (one order of magnitude), which supports the hypothesis that wines bioactivity is contributed mainly to polar lipids while the contribution of neutral lipids is rather small. The most potent wines were the red wine Cabernet Sauvignon (main variety Cabernet Sauvignon) and the white wine Ambelon (main variety Rompola) demonstrating that the variable of grape is important for the biological activity and not the color. ${ }^{97}$ In an effort to examine the origin of the active compounds, the lipids of the respective musts of these two wines were extracted. Two interesting points arose from these data: first, total neutral lipids of musts exerted a strong inhibitory activity attaining approximately the same order of magnitude with total lipids and total polar lipids; second, polar lipids of white grape must were more potent inhibitors (one order of magnitude) than of the red grape, but after fermentation red wine with corresponding lipids possessed the same activity as white wine lipids. The above data indicate that the biological activity in the white wine remained practically unchanged before and after fermentation, while red wine seems to improve its health value during fermentation.

The above results bring out the antiatherogenic properties of the Greek white variety Rompola and support the theory that the variety of the grape and the type of phenolics in the final product (the wine) is determinant for its biological effects. Some of the most potent molecules identified after chromatographic purification and electrospray mass spectrometry (ESMS) are presented in Figure 2. ${ }^{92,98,99}$ It should be noted that less than one glass of wine (approximately $110 \mathrm{~mL}$ ) or less than $10 \mathrm{~mL}$ of must contain the effective concentrations of all the aforementioned bioactive compounds. As shown in Figure 2, apart from the phenolic compounds, classical phospho- and glyco-glycerolipids are also present in wine and exhibit important biological actions. ${ }^{98,99}$ It is also important to mention that the action of these compounds is blocked by the specific PAF-inhibitor, BN52021, indicating that these compounds act partly through the PAF-R.

\section{Angiogenesis}

Angiogenesis is also involved in the pathogenesis of several disorders such as tumor growth, atherosclerosis, and proliferative retinopathies. ${ }^{100}$ During this multistep process, capillary endothelial cells (EC) migrate and proliferate in order to form a three-dimensional structure capable of carrying blood, resulting in part from the concerted action of EC-directed growth factors. VEGF as well as other angiogenic factors are capable of inducing the production of $\mathrm{PAF}^{62,101}$ from EC and subsequently promote EC shape changes, migration, proliferation, and angiogenesis. Among them sphingolipid derivative: sphingosine 1-phosphate (S1P) plays an important role in the regulation of EC migration and proliferation which are prerequisites of the angiogenic process. ${ }^{102}$ In a recent study, red grape skin polyphenolic extract has been found to inhibit angiogenesis by decreasing S1P and VEGF-induced PAF synthesis and its subsequent chemotactic effect on bovine aortic endothelial cells. ${ }^{102}$

\section{Actions on PAF metabolism}

PAF levels are determined by the balance between its synthesis and its degradation ${ }^{103}$ (Figure 3 ). The remodeling pathway involves a structural modification of pre-existing ether-linked choline-containing phospholipids that serve as structural components of membranes. Following cell stimulation, cPLA $_{2}$ is activated, becomes membrane-associated, and hydrolyzes the fatty acyl moiety esterified at the sn-2 position of membrane phospholipids. The 2-lyso-phospholipids (lyso-PC) that are generated serve as substrates for lyso-PAF acetyltransferase (lyso-PAF-AT), an enzyme that transfers an acetyl group from acetyl-CoA to lyso-PC yielding PAF. The de novo pathway entails a specific stepwise sequence of reactions beginning with the acetylation and then the dephosphorylation of 1-O-alkyl-sn-glycero-3-phosphate, which is an ether-linked metabolic intermediate formed soon after the ether bond is created. These reactions are sequentially catalyzed by an acetyl-CoA:alkyl-lyso-glycerophosphate acetyltransferase (ALPA-AT) and an alkylacetyl glycero-phosphate phosphohydrolase, respectively. The 1-O-alkyl-2-acetyl-glycerol is converted to PAF by a specific dithiothreitol-insensitive CDP-choline:1-alkyl-2acetyl-sn-glycerol cholinephosphotransferase (PAF-CPT). The main degrading enzyme of PAF is PAF-acetylhydrolase (PAF-AH), which in serum is found, bound to LDL and in less extent in high-density lipoprotein (HDL). Its location is the reason for the new name of this enzyme, lipoproteinassociated phospholipase $\mathrm{A}_{2}\left(\mathrm{LpPLA}_{2}\right)$. LpPLA $\mathrm{A}_{2}$ have drawn a lot of the scientific interest during recent years and nowadays is considered as a new biochemical marker for CVDs.

Flavonoids inhibition of $\mathrm{PLA}_{2}$ activity has been demonstrated in a number of in vitro studies. ${ }^{104,105}$ In addition, flavonoids have been found to inhibit lyso-PAF AT and this inhibition depends on the structure of the flavonoid. More specifically, luteolin exhibited the strongest inhibitory effect on lyso-PAF AT in the cell-free system, followed by quercetin, while the structurally related flavonoids, apigenin and keampferol, showed only a weak inhibitory action, 

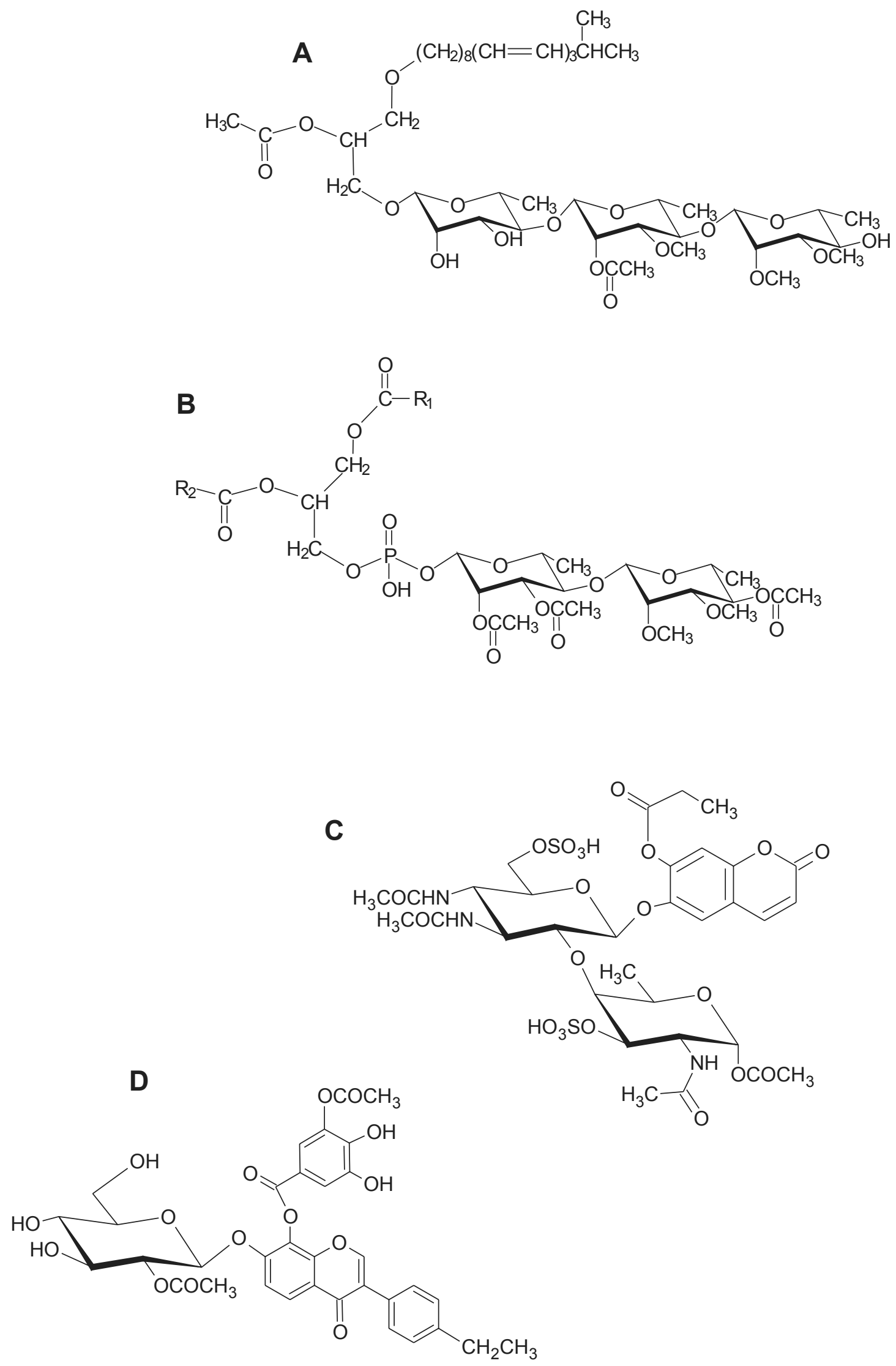

Figure 2 Structures of platelet-activating factor antagonists isolated from wines. A) isolated from white wine (Ambelon, Robola);B) isolated from white must (Robola);C) isolated from white wine (Ambelon, Robola); D) isolated from red wine (Cabernet Sauvignon). 
which suggests that the 5'-hydroxyl group is important for the inhibitory action. In addition, saturation of the $\mathrm{C} 2-3$ bond of luteolin (producing eriodictyol) prevented inhibition of lyso-PAF-AT activity, which suggests a requirement for planarity of the heterocyclic ring. $O$-Glycosylation at the C-7 position (luteolin-7-glucoside) also abolished the inhibitory activity, but the reason for this is still unknown. ${ }^{106}$ Moreover, quercetin, naringin, and hesperedin have been found to modulate PAF metabolism in endothelial cells during oxidative stress. The above flavonoids blocked PAF production through inhibition of lyso-PAF-AT and PLA And $_{2}$ ancomitantly by enhancing the formation of less biologically active lipid mediators through the PAF-dependent CoA independent transacetylase (TA). ${ }^{107}$ This enzyme hydrolyzes PAF, producing acyl-PAF, which has an inhibitory role in the initiation and progression of atherosclerosis. The activity of PAF-AH was not affected.

\section{Bioavailability}

On the other hand, it should be noted that evaluating the absorption and bioavailability of phenolic compounds is critical and should be completed before the evaluation of their biological activity or their potential nutritional value. Several studies have confirmed that some of these compounds are absorbed in sufficiently high concentration in order to have physiological effects. In addition chemical modifications such as glycosylation, methylation, glucuronidation, sulfation, or acetylation may also happen during absorption and the

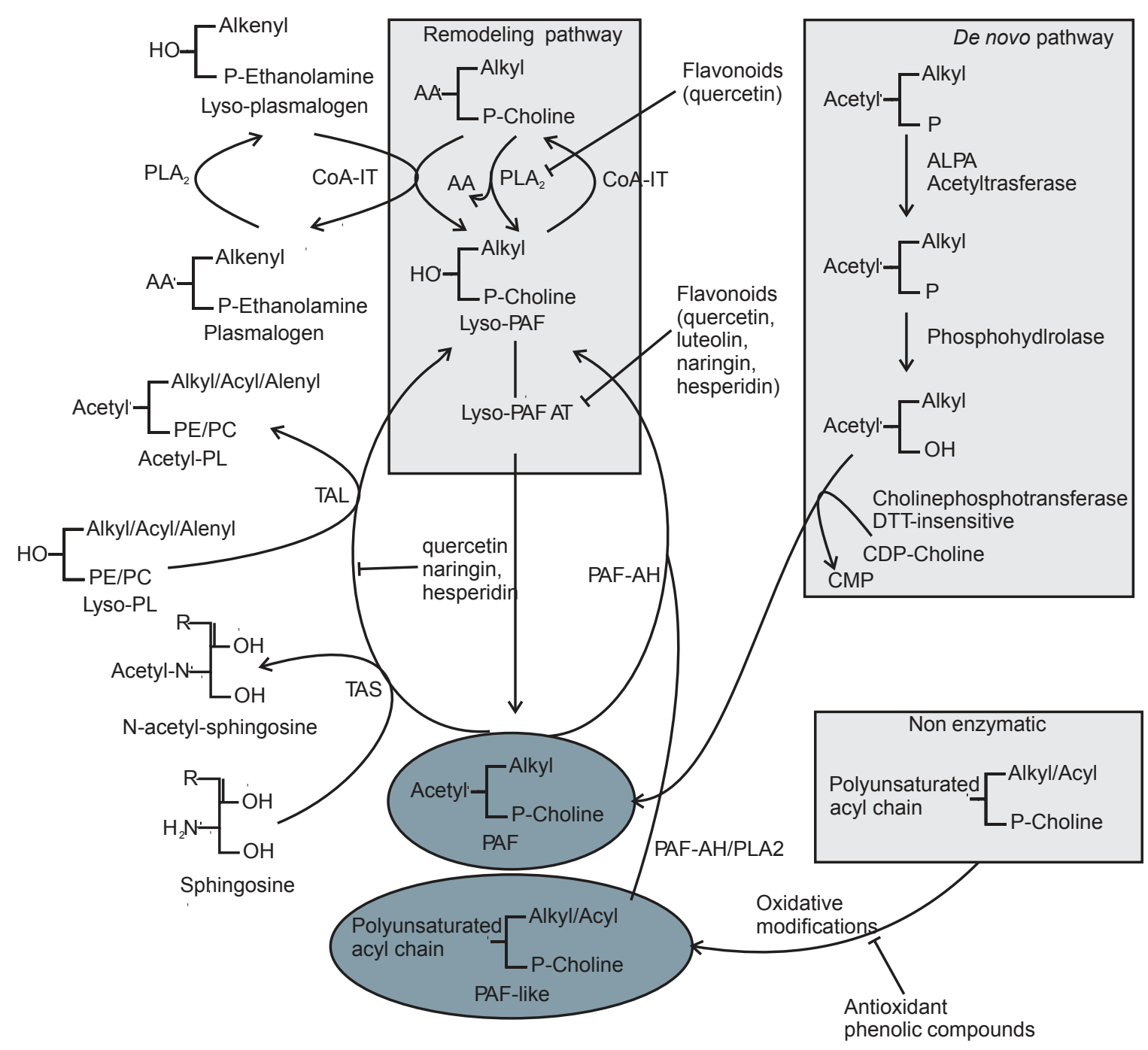

Figure 3 The pathways of PAF biosynthesis and catabolism. The possible points of its production inhibition by wine phenolics are presented.

Abbreviations: CDP, cytidine 5'-diphosphate; CoA-IT, coenzyme A-independent transacylase; DTT, dithiothreitol; lyso-PC, lysophosphatidylcholine; PAF, platelet-activating factor; PAF-AH, PAF-acetylhydrolase; PL, phospholipid; PLA, cytosolic phospholipase A; ; $_{2}$, AL, PAF-dependent transacetylase to lysophospholipids; TAS, PAF-dependent transacetylase to sphingosine. 
bioactivity of these derivatives should be evaluated. Among several phenolics, the bioavailability of anthocyanins, quercetin, resveratrol, and epicatechin from dietary sources has already been reported. ${ }^{108,109}$ Studies in humans have shown that some polyphenols from red wine are absorbed and are bound to LDL particles following red wine ingestion. ${ }^{110}$ However, improvements in high-pressure liquid chromatography and gas chromatography-mass spectrometry methodologies are required in order to measure phenolic compounds or their metabolites in plasma and urine.

\section{Conclusions}

Atherosclerosis is a multifactorial inflammatory disease and is considered the most important underlying cause of many CVDs. Although the essential steps have been recognized, the details that encode each one are not fully elucidated yet. However, it is widely accepted that some mediators play a crucial role in the initiation and the progression of atherogenesis. Among them PAF and oxidized phospolipids play an essential role in the initiation and prolongation of inflammation as we have previously proposed. ${ }^{31}$

Diet is considered as an important risk factor that could either promote health or increase risk of chronic disease. ${ }^{1-3}$ Although an unhealthy diet is associated with increase incidence of various diseases such as CVDs and cancer, there are some notable exceptions from the above and several paradoxes appear. The term "paradox" refers to the correlation of a high-fat diet with a lower incidence of CHD found in Mediterranean cultures in contrast to a higher incidence of CHD among most Western cultures. The highlight is the French paradox although other terms such as North-South paradox or Mediterranean paradox could also be used. ${ }^{9,11}$ Nevertheless, the most difficult issue is not the description but the explanation of these paradoxes taking also under consideration that some studies bring into question the positive association of fat intake with the risk of cardiovascular events. ${ }^{111}$

The Mediterranean diet is considered to be a pattern of healthy diet that promotes human health. We have demonstrated that several Mediterranean foods, such as olive oil, olive pomace, fish, honey, and traditional Greek meals contain PAF inhibitors/antagonists that could reduce its actions. ${ }^{31}$ Moreover, we have confirmed in in vivo experiments that the consumption of foods rich in PAF inhibitors/antagonists could improve health parameters. More specifically, olive oil and olive pomace polar extracts reduced the atheromatic lesion in hypercholesterolemic rabbits. ${ }^{112,113}$ In addition, a study of diabetic mellitus patients revealed that an one month diet consisting of several traditional foods was capable of reducing the platelet sensitivity of this group compared with the control group. ${ }^{114}$

Among the already known and well characterized biological effects of wine bioactive compounds, we would like to highlight the co-existence of compounds that could modulate the production and the actions of PAF. PAF is capable of causing increased endothelial cell permeability and is an essential mediator for the adhesion and the infiltration of leukocytes into subendothelial space. Moreover, it causes platelet activation and aggregation as well as SMC proliferation and migration. Phenolics reduce the oxidation of LDL particles and in turn the production of PAF and PAF-like lipids. Moreover phenolics such as reveratrol and flavonoids have been shown to reduce PAF enzymatic production and therefore leading to lower PAF levels. Several phenolics, including resveratrol, and wine extracts inhibit PAF-induced platelet aggregation and resveratrol also prevent $\mathrm{PAF}$-induced leukocyte recruitment and endothelial barrier disruption. Considering the crucial role of PAF in the initiation and prolongation of inflammation the consumption of a diet rich in compounds that downregulate PAF-production, and/or enhance PAFdegradation, and/or inhibit its actions, would be beneficial for health.

The aforementioned data shed further light on the nature of the beneficial effects of moderate wine consumption since it contains a significant number of lipids including phenolics with antithrombotic as well as antiatherogenetic actions. These data, in correlation with our proposed mechanism of atheromatosis generation, may offer a new insight into the well known "French paradox" and expand the already reported mechanisms by including the inhibition of PAF actions.

\section{Disclosure}

The authors report no conflicts of interest in this work.

\section{References}

1. Keys A. Coronary heart disease in seven countries. 1970. Nutrition. 1997;13(3):250-252.

2. Bazzano LA, He J, Ogden LG, et al. Fruit and vegetable intake and risk of cardiovascular disease in US adults: the first National Health and Nutrition Examination Survey Epidemiologic Follow-up Study. Am J Clin Nutr. 2002;76(1):93-99.

3. Liu S, Lee IM, Ajani U, Cole SR, Buring JE, Manson JE. Intake of vegetables rich in carotenoids and risk of coronary heart disease in men: The Physicians' Health Study. Int J Epidemiol. 2001;30(1):130-135.

4. Kok FJ, Kromhout D. Atherosclerosis - epidemiological studies on the health effects of a Mediterranean diet. Eur J Nutr. 2004;43(Suppl 1):I/2-5. 
5. Verschuren WM, Jacobs DR, Bloemberg BP, et al. Serum total cholesterol and long-term coronary heart disease mortality in different cultures. Twenty fiveyear follow-up of the seven countries study. JAMA. 1995;274(2):131-136.

6. Keys A. Seven Countries. A multivariate analysis of death and coronary heart disease. A Commonwealth Fund Book. Cambridge: Harvard University Press, 1980. p. 1-381.

7. de Lorgeril M, Renaud S, Mamelle N, et al. Mediterranean alphalinolenic acid-rich diet in secondary prevention of coronary heart disease. Lancet. 1994;343:1454-1459.

8. Singh RB, Dubnov G, Niaz MA, et al. Effect of an Indo-Mediterranean diet on progression of coronary artery disease in high risk patients (Indo-Mediterranean diet heart study): a randomised single-blind trial. Lancet. 2002;360:1455-1461.

9. Renaud S, de Lorgeril M, Delaye J, et al. Cretan Mediterranean diet for prevention of coronary heart disease. Am J Clin Nutr. 1995;61 (6 Suppl):1360S-1367S

10. Panagiotakos DB, Pitsavos C, Arvaniti F, et al. Adherence to the Mediterranean food pattern predicts the prevalence of hypertension, hypercholesterolemia, diabetes and obesity, among healthy adults; the accuracy of the MedDietScore. Prev Med. 2007;44(4):335-340.

11. Renaud S, de Lorgeril M. Wine, alcohol, platelets, and the French paradox for coronary heart disease. Lancet. 1992;339:1523-1526.

12. McMichael J. French wine and death certificates. Lancet. 1979; 1:1186-1187.

13. Law M, Wald N. Why heart disease mortality is low in France: the time lag explanation. BMJ. 1999;318(7196):1471-1476.

14. de Lorgeril M, Salen P, Paillard F, Laporte F, Boucher F, de Leiris J. Mediterranean diet and the French paradox: two distinct biogeographic concepts for one consolidated scientific theory on the role of nutrition in coronary heart disease. Cardiovasc Res. 2002;54(3):503-515.

15. St Leger AS, Cochrane AL, Moore F. Factors associated with cardiac mortality in developed countries with particular reference to the consumption of wine. Lancet. 1979;1(8124):1017-1020.

16. Tunstall-Pedoe H, Kuulasmaa K, Mahonen M, et al. Contribution of trends in survival and coronary-event rates to changes in coronary heart disease mortality: 10-year results from 37 WHO MONICA Project populations. Lancet. 1999;353:1547-1557.

17. Kuulasmaa K, Tunstall-Pedoe H, Dobson A, et al. Estimation of contribution of changes in classic risk factors to trends in coronary-event rates across the MONICA Project populations. Lancet. 2000;355: 675-687.

18. Klatsky AL, Armstrong MA, Friedman GD. Red wine, white wine, liquor, beer, and risk for coronary artery disease hospitalization. $\mathrm{Am} \mathrm{J}$ Cardiol. 1997;80:416-420.

19. Rimm EB. Invited commentary - Alcohol consumption and coronary heart disease: Good habits may be more important than just good wine. Am J Epidemiol. 1996;143:1094-1098.

20. Criqui MH, Ringel BL. Does diet or alcohol explain the French paradox? Lancet. 1994;344(8939-8940):1719-1723.

21. Klatsky AL, Friedman GD, Armstrong MA, Kipp H. Wine, liquor, beer, and mortality. Am J Epidemiol. 2003;158(6):585-595.

22. Grønbaek M, Deis A, Sørensen TI, Becker U, Schnohr P, Jensen G Mortality associated with moderate intakes of wine, beer, or spirits. BMJ. 1995;310(6988):1165-1169.

23. Truelsen T, Gronbaek M, Schnohr P, Boysen G. Intake of beer, wine, and spirits and risk of stroke: the Copenhagen city heart study. Stroke. 1998;29(12):2467-2472.

24. Renaud SC, Guéguen R, Siest G, Salamon R. Wine, beer, and mortality in middle-aged men from eastern France. Arch Intern Med. 1999;159(16): 1865-1870.

25. Grønbaek M, Becker U, Johansen D, et al. Type of alcohol consumed and mortality from all causes, coronary heart disease, and cancer. Ann Intern Med. 2000;133(6):411-419.

26. Di Castelnuovo A, Rotondo S, Iacoviello L, Donati MB, De Gaetano G. Meta-analysis of wine and beer consumption in relation to vascular risk. Circulation. 2002;105(24):2836-2844.

27. Lamuela-Raventós RM, de la Torre-Boronat MC. Beneficial effects of white wines. Drugs Exp Clin Res. 1999;25(2-3):121-124.
28. Wallerath T, Poleo D, Li H, Förstermann U. Red wine increases the expression of human endothelial nitric oxide synthase: a mechanism that may contribute to its beneficial cardiovascular effects. $J$ Am Coll Cardiol. 2003;41(3):471-478.

29. Vogel RA. Vintners and vasodilators: are French red wines more cardioprotective? J Am Coll Cardiol. 2003;41(3):479-481.

30. Demopoulos CA, Pinckard RN, Hanahan DJ. Platelet-activating factor. Evidence for 1-O-alkyl-2-acetyl-sn-glyceryl-3-phosphorylcholine as the active component (a new class of lipid chemical mediators). J Biol Chem. 1979;254:9355-9358.

31. Demopoulos CA, Karantonis HC, Antonopoulou S. Platelet activating factor - a molecular link between atherosclerosis theories. Eur J Lipid Sci Technol. 2003;105:705-716.

32. Honda Z, Nakamura M, Miki I, et al. Cloning by functional expression of platelet-activating factor receptor from guinea-pig lung. Nature. 1991;349:342-346.

33. Marathe GK, Prescott SM, Zimmerman GA, McIntyre TM. Oxidized LDL contains inflammatory PAF-like phospholipids. Trends Cardiovasc Med. 2001;11:139-142.

34. Androulakis N, Durand H, Ninio E, Tsoukatos DC. Molecular and mechanistic characterization of platelet-activating factor-like bioactivity produced upon LDL oxidation. J Lipid Res. 2005;46(9): 1923-1932.

35. Liapikos TA, Antonopoulou S, Karabina SP, et al. Platelet-activating factor formation during oxidative modification of low-density lipoprotein when PAF-acetylhydrolase has been inactivated. BBA-Lipid Lipid Metab. 1994;1212:353-360.

36. Braquet P, Hosford D, Koltz P, Guilbaud J, Paubert-Braquet M. Effect of platelet-activating factor on tumor necrosis factor-induced superoxide generation from human neutrophils. Possible involvement of $\mathrm{G}$ proteins. Lipids. 1991;26(12):1071-1075.

37. Bautista AP, Spitzer JJ. Platelet activating factor stimulates and primes the liver, Kupffer cells and neutrophils to release superoxide anion. Free Radic Res Commun. 1992;17(3):195-209.

38. Gaut JP, Heinecke JW. Mechanisms for oxidizing low-density lipoprotein. Insights from patterns of oxidation roducts in the artery wall and from mouse models of atherosclerosis. Trends Cardiovasc Med. 2001;11(3-4):103-112.

39. Heller R, Bussolino F, Ghigo D, et al. Human endothelial cells are target for platelet activating factor. Platelet activating factor induces platelet activating factor synthesis in human umbilical vein endothelial cells. J Immunol. 1992;149:3682-3688.

40. Handley DA, Arbeeny CM, Lee ML, Van Valen RG, Saunders RN. Effect of platelet activating factor on endothelial permeability to plasma macromolecules. Immunopharmacology. 1984;8(3-4): 137-142.

41. Jiang Y, Wen K, Zhou X, Schwegler-Berry D, Castranova V, He P. Three-dimensional localization and quantification of PAF-induced gap formation in intact venular microvessels. Am J Physiol Heart Circ Physiol. 2008;295(2):H898-H906.

42. Sánchez FA, Kim DD, Durán RG, Meininger CJ, Durán WN. Internalization of eNOS via caveolae regulates PAF-induced inflammatory hyperpermeability to macromolecules. Am J Physiol Heart Circ Physiol. 2008;295(4):H1642-H1648.

43. Springer TA. Traffic signals for lymphocyte recirculation and leukocyte emigration: the multistep paradigm. Cell. 1994;76:301-314.

44. McMeekin SR, Dransfield I, Rossi AG, Haslett C, Walker TR. E-selectin permits communication between PAF receptors and TRPC channels in human neutrophils. Blood. 2006;107(12):4938-4945.

45. Kuijpers TW, Hakkert BC, Hart MH, Roos D. Neutrophil migration across monolayers of cytokine-prestimulated endothelial cells: a role for platelet-activating factor and IL-8. J Cell Biol. 1992;117(3): 565-572.

46. Zimmerman GA, McIntyre TM, Prescott SM, Stafforini DM. The plateletactivating factor signaling system and its regulators in syndromes of inflammation and thrombosis. Crit Care Med. 2002;30(5 Suppl):S294-301.

47. Weyrich AS, McIntyre TM, McEver RP, Prescott SM, Zimmerman GA Monocyte tethering by P-selectin regulates monocyte chemotactic protein-1 and tumor necrosis factor-alpha secretion. Signal integration and NF-kappa B translocation. J Clin Invest. 1995;95(5): 2297-2303. 
48. McManus LM, Hanahan DJ, Pinckard RN. Human platelet stimulation by acetyl glyceryl ether phosphorylcholine. J Clin Invest. 1981;67(3): 903-906.

49. Golino P, Ambrosio G, Ragni M, et al. Short-term and long-term role of platelet activating factor as a mediator of in vivo platelet aggregation. Circulation. 1993;88(3):1205-1214.

50. Rimm EB, Williams P, Fosher K, Criqui M, Stampfer MJ. Moderate alcohol intake and lower risk of coronary heart disease: meta-analysis of effects on lipids and haemostatic factors. BMJ. 1999;319(7224): $1523-1528$.

51. Waterhouse AL. Wine phenolics. Ann N Y Acad Sci. 2002;957: 21-36.

52. Dell'Agli M, Buscialà A, Bosisio E. Vascular effects of wine polyphenols. Cardiovasc Res. 2004;63(4):593-602.

53. Cordova AC, Jackson LS, Berke-Schlessel DW, Sumpio BE. The cardiovascular protective effect of red wine. J Am Coll Surg. 2005;200(3):428-439.

54. Howard A, Chopra M, Thurnham D, Strain J, Fuhrman B, Aviram M. Red wine consumption and inhibition of LDL oxidation: what are the important components? Med Hypotheses. 2002;59(1):101-104.

55. Cui J, Tosaki A, Cordis GA, et al. Cardioprotective abilities of white wine. Ann N Y Acad Sci. 2002;957:308-316.

56. Bertelli AAE, Migliori M, Panichi V, et al. Oxidative stress and inflammatory reaction modulation by white wine. Ann NY Acad Sci. 2002;957:295-301.

57. Va'zquez-Agell M, Sacanella E, Tobias E, et al. Inflammatory markers of atherosclerosis are decreased after moderate consumption of cava (sparkling wine) in men with low cardiovascular risk. $J$ Nutr. 2007;137(10):2279-2284.

58. Li H, Förstermann U. Nitric oxide in the pathogenesis of vascular disease. J Pathol. 2000;190:244-254.

59. Vandenbroucke E, Mehta D, Minshall R, Malik AB. Regulation of endothelial junctional permeability. Ann N Y Acad Sci. 2008;1123: 134-145.

60. Mariano F, Bussolati B, Migliori M, Russo S, Triolo G, Camussi G. Platelet-activating factor synthesis by neutrophils, monocytes, and endothelial cells is modulated by nitric oxide production. Shock. 2003;19(4):339-344.

61. Kikuchi M, Shirasaki H, Himi T. Platelet-activating factor (PAF) increases NO production in human endothelial cells-real-time monitoring by DAR-4M AM. Prostaglandins Leukot Essent Fatty Acids. 2008;78(4-5):305-309.

62. Brkovic A, Sirois MG. Vascular permeability induced by VEGF family members in vivo: role of endogenous PAF and $\mathrm{NO}$ synthesis. $J$ Cell Biochem. 2007;100(3):727-737.

63. Ferrara N. Molecular and biological properties of vascular endothelial growth factor. J Mol Med. 1999;77(7):527-543.

64. Feng AN, Chen YL, Chen YT, Ding YZ, Lin SJ. Red wine inhibits monocyte chemotactic protein-1 expression and modestly reduces neointimal hyperplasia after balloon injury in cholesterol-fed rabbits. Circulation. 1999;100:2254-2259.

65. Stoclet JC, Kleschyov A, Andriambeloson E, Diebolt M, Andriantsitohaina R. Endothelial no release caused by red wine polyphenols. J Physiol Pharmacol. 1999;50(4):535-540.

66. Dudley JI, Lekli I, Mukherjee S, Das M, Bertelli AA, Das DK. Does white wine qualify for French paradox? Comparison of the cardioprotective effects of red and white wines and their constituents: resveratrol, tyrosol, and hydroxytyrosol. J Agric Food Chem. 2008;56(20):9362-9373.

67. Wallerath T, Deckert G, Ternes T, et al. Resveratrol, a polyphenolic phytoalexin present in red wine, enhances expression and activity of endothelial nitric oxide synthase. Circulation. 2002;106(13): 1652-1658.

68. Shigematsu S, Ishida S, Hara M, et al. Resveratrol, a red wine constituent polyphenol, prevents superoxide-dependent inflammatory responses induced by ischemia/reperfusion, platelet-activating factor, or oxidants. Free Radic Biol Med. 2003;34(7):810-817.
69. Csiszar A, Smith K, Labinskyy N, Orosz Z, Rivera A, Ungvari Z. Resveratrol attenuates TNF-alpha-induced activation of coronary arterial endothelial cells: role of NF-kappaB inhibition Am J Physiol Heart Circ Physiol. 2006;291(4):H1694-1699.

70. Lee $\mathrm{G}, \mathrm{Na} \mathrm{HJ}$, Namkoong $\mathrm{S}$, et al. 4-O-methylgallic acid down-regulates endothelial adhesion molecule expression by inhibiting NF-kappaB-DNA-binding activity. Eur J Pharmacol. 2006;551(1-3):143-151.

71. Murase T, Kume N, Hase T, et al. Gallates inhibit cytokine-induced nuclear translocation of NF-kappaB and expression of leukocyte adhesion molecules in vascular endothelial cells. Arterioscler Thromb Vasc Biol. 1999;19(6):1412-1420.

72. Kaneider NC, Mosheimer B, Reinisch N, Patsch JR, Wiedermann CJ. Inhibition of thrombin-induced signaling by resveratrol and quercetin: effects on adenosine nucleotide metabolism in endothelial cells and platelet-neutrophil interactions. Thromb Res. 2004;114(3): 185-194.

73. Sneddon AA, McLeod E, Wahle KW, Arthur JR. Cytokine-induced monocyte adhesion to endothelial cells involves platelet-activating factor: suppression by conjugated linoleic acid. Biochim Biophys Acta. 2006;1761(7):793-801.

74. Jeong YJ, Choi YJ, Choi JS, et al. Attenuation of monocyte adhesion and oxidised LDL uptake in luteolin-treated human endothelial cells exposed to oxidised LDL. Br J Nutr. 2007;97(3):447-457.

75. Araim O, Ballantyne J, Waterhouse A, Sumpio BE. Inhibition of vascular smooth muscle cell proliferation with red wine and red wine polyphenols. J Vasc Surg. 2002;35:1226-1232.

76. Imai Y, Clemmons DR. Roles of phosphatidylinositol-3-kinase and mitogen-activated protein kinase pathways in stimulation of vascular smooth muscle cell migration and deoxyribonucleic acid synthesis by insulin-like growth factor-I. Endocrinology. 1999;140(9):4228-4235.

77. Hedges JC, Dechert MA, Yamboliev IA, et al. A role for p38(MAPK)/ HSP27 pathway in smooth muscle cell migration. J Biol Chem. 1999;274:24211-24219.

78. Stoll LL, Spector AA. Interaction of platelet-activating factor with endothelial and vascular smooth muscle cells in coculture. $J$ Cell Physiol. 1989;39:253-261.

79. Stengel D, O’Neil C, Brochériou I, et al. Receptor is preferentially expressed in a distinct synthetic phenotype of smooth muscle cells cloned from human internal thoracic artery: functional implications in cell migration. Biochem Biophys Res Commun. 2006;346(3): 693-699.

80. Wara AK, Mitsumata M, Yamane T, Kusumi Y, Yoshida Y. Gene expression in endothelial cells and intimal smooth muscle cells in atherosclerosis-prone or atherosclerosis-resistant regions of the human aorta. J Vasc Res. 2008;45(4):303-313.

81. Heery JM, Kozak M, Stafforini DM, et al. Oxidatively modified LDL contains phospholipids with platelet-activating factor-like activity and stimulates the growth of smooth muscle cells. J Clin Invest. 1995;96(5):2322-2330.

82. Akiba S, Yamaguchi H, Kumazawa S, Oka M, Sato T. Suppression of oxidized LDL-induced PDGF receptor beta activation by ginkgo biloba extract reduces MMP-1 production in coronary smooth muscle cells. J Atheroscler Thromb. 2007;14(5):219-225.

83. Stiko-Rahm A, Hultgårdh-Nilsson A, Regnström J, Hamsten A, Nilsson J. Native and oxidized LDL enhances production of PDGF AA and the surface expression of PDGF receptors in cultured human smooth muscle cells. Arterioscler Thromb. 1992;12(9): 1099-1109.

84. Iijima K, Yoshizumi M, Ouchi Y, et al. Red wine polyphenols inhibit proliferation of vascular smooth muscle cells and downregulate expression of cyclin A gene. Circulation. 2000;101: 805-811.

85. Iijima K, Yoshizumi M, Hashimoto M, et al. Red wine polyphenols inhibit vascular smooth muscle migration through two distinct signaling pathways. Circulation. 2002;105:2404-2410.

86. Oak MH, Chataigneau M, Keravis T, et al. Red wine polyphenolic compounds inhibit vascular endothelial growth factor expression in vascular smooth muscle cells by preventing the activation of the $\mathrm{p} 38$ mitogen-activated protein kinase pathway. Arterioscler Thromb Vasc Biol. 2003;23:1001-1007. 
87. Ruf JC. Wine and polyphenols related to platelet aggregation and atherothrombosis. Drugs Exp Clin Res. 1999;25(2-3):125-131.

88. Pignatelli P, Pulcinelli FM, Celestini A, et al. The flavonoids quercetin and catechin synergistically inhibit platelet function by antagonizing the intracellular production of hydrogen peroxide. Am J Clin Nutr. 2000;72(5):1150-1155

89. Gryglewski RJ, Korbut R, Robak J, Swies J. On the mechanism of antithrombotic action of flavonoids. Biochem Pharmacol. 1987;36(3):317-322.

90. Landolfi R, Mower RL, Steiner M. Modification of platelet function and arachidonic acid metabolism by bioflavonoids. Structure-activity relations. Biochem Pharmacol. 1984;33(9):152515-152530.

91. Fragopoulou E, Nomikos T, Karantonis HC, et al. Biological activity of acetylated phenolic compounds. J Agric Food Chem. 2007;55(1): $80-89$.

92. Fragopoulou E, Nomikos T, Antonopoulou S, Mitsopoulou CA, Demopoulos CA. Separation of biologically active lipids from red wine. J Agric Food Chem. 2000;48(4):1234-1238.

93. Tzeng SH, Ko WC, Ko FN, Teng CM. Inhibition of platelet aggregation by some flavonoids. Thromb Res. 1991;64(1):91-100.

94. Pace-Asciak CR, Hahn S, Diamandis EP, Soleas G, Goldberg DM. The red wine phenolics trans-resveratrol and quercetin block human platelet aggregation and eicosanoid synthesis: implications for protection against coronary heart disease. Clin Chim Acta. 1995;235(2): 207-219.

95. Pace-Asciak CR, Rounova O, Hahn SE, Diamandis EP, Goldberg DM. Wines and grape juices as modulators of platelet aggregation in healthy human subjects. Clin Chim Acta. 1996;246(1-2):163-182.

96. Rein D, Paglieroni TG, Pearson DA, et al. Cocoa and wine polyphenols modulate platelet activation and function. J Nutr. 2000;130 (8S Suppl):2120S-2126S

97. Fragopoulou E, Nomikos T, Tsantila N, Mistopoulou A, Zabetakis I, Demopoulos CA. Biological activity of total lipids from red and white wine/must. J Agric Food Chem. 2001;49(11):5186-5193.

98. Fragopoulou E, Antonopoulou S, Nomikos T, Demopoulos CA. Structure elucidation of phenolic compounds from red/white wine with antiatherogenic properties. Biochim Biophys Acta. 2003;1632(1-3): 90-99.

99. Fragopoulou E, Antonopoulou S, Demopoulos CA. Biologically active lipids with antiatherogenic properties from white wine and must. J Agric Food Chem. 2002;50(9):2684-2694.

100. Carmeliet P. Mechanisms of angiogenesis and arteriogenesis. Nat Med. 2000;6(4):389-395.
101. Maliba R, Lapointe S, Neagoe PE, Brkovic A, Sirois MG. Angiopoietins- 1 and -2 are both capable of mediating endothelial PAF synthesis: intracellular signalling pathways. Cell Signal. 2006;18(11): 1947-1957.

102. Barthomeuf C. Inhibition of S1P-induced angiogenesis, metastasis and inflammation by dietary polyphenols. Free Radic Biol Med. 2007;42(2):312-313.

103. Snyder F. Platelet-activating factor: the biosynthetic and catabolic enzymes. Biochem J. 1995;305(Pt 3):689-705.

104. Lindahl M, Tagesson C. Selective inhibition of group II phospholipase A2 by quercetin. Inflammation. 1993;17(5):573-582.

105. Gil B, Sanz MJ, Terencio MC, et al. Effects of flavonoids on Naja naja and human recombinant synovial phospholipases $\mathrm{A} 2$ and inflammatory responses in mice. Life Sci. 1994;54(20):L333-L338.

106. Yanoshita R, Chang HW, Son KH, Kudo I, Samejima Y. Inhibition of lysoPAF acetyltransferase activity by flavonoids. Inflamm Res. 1996;45(11):546-549.

107. Balestrieri ML, Castaldo D, Balestrieri C, Quagliuolo L, Giovane A, Servillo L. Modulation by flavonoids of PAF and related phospholipids in endothelial cells during oxidative stress. J Lipid Res. 2003;44(2): 380-387.

108. Cao G, Prior A. Anthocyanins are detected in humanbplasma after oral administration of an elderberry extract. Clin Chem. 1999;45:574-576.

109. Manach C, Morand C, Crespy V, et al. Quercetin is recovered in human plasma as conjugated derivative which retain antioxidant properties. FEBS Lett. 1998;426:331-336.

110. Nigdikar S, Williams NR, Griffin BA, Howard AN. Consumption of red wine polyphenols reduces the susceptibility of low-density lipoproteins to oxidation. Am J Clin Nutr. 1998;68:258-265.

111. Leosdottir M, Nilsson PM, Nilsson JA, Berglund G. Cardiovascular event risk in relation to dietary fat intake in middle-aged individuals: data from The Malmö Diet and Cancer Study. Eur J Cardiovasc Prev Rehabil. 2007;14(5):701-706.

112. Karantonis HC, Antonopoulou S, Perrea DN, et al. In vivo antiatherogenic properties of olive oil and its constituent lipid classes in hyperlipidemic rabbits. Nutr Metab Cardiovasc Dis. 2006;16(3): 174-185.

113. Tsantila N, Karantonis HC, Perrea DN, et al. Antithrombotic and antiatherosclerotic properties of olive oil and olive pomace polar extracts in rabbits. Mediators Inflamm. 2007;2007:36204.

114. Antonopoulou S, Fragopoulou E, Karantonis HC, et al. Effect of traditional Greek Mediterranean meals on platelet aggregation in normal subjects and in patients with type 2 diabetes mellitus. $J$ Med Food. 2006;9(3):356-362. 
